\title{
Effectiveness of PEEK Framework in Comparison to Metal Framework for Fixed Dental Prosthesis: A Systematic Review
}

\author{
Rupawat D Kamlesh ${ }^{1}$, Deepak Nallaswamy², Dhanraj Ganapathy ${ }^{3}$
}

\begin{abstract}
Aim: This systematic review was done to evaluate Polyether Ether Ketone (PEEK) over metal-ceramic alloy as a fixed partial denture framework. Materials and methods: This review adhered to the PRISMA guidelines for conducting and reporting systematic reviews and meta-analyses. Three independent reviewers screened the literature pertinent to PEEK in fixed dental prosthetic applications with time limits from the years 1995 January to 2020 January. The search strategy was done based on PICO formulation. PICO analysis was used to create a search questionnaire, and a complete search was piloted in PubMed, Cochrane, Embase, Ovid, Science Direct databases for related manuscripts. Following screening and final selection, all the relevant information from the articles was extracted, tabulated, analyzed, and interpreted.

Results: 140 papers were rejected grounded on the title and the abstract from the 165 publications found after perusing all databases. Twenty papers were eliminated based on our inclusion and exclusion criteria, leaving only five researches to be included based on their central data. Meta-analysis could not be initiated due to the heterogeneity of the selected articles.

Conclusion: Based on this systematic review, PEEK-fixed partial dentures are reported to show better esthetics and despite the fact, PEEK frameworks showed equivalent mechanical properties as metal alloys, there is insufficient evidence to validate superior mechanical properties compared to its metal counterpart. Hence future studies are required in this field for further understanding and authentication.

Clinical significance: PEEK can be effectively used as an appropriate superstructure for dental implants, temporary abutments, implant aided bars, framework for removable prostheses, and fixed dental prostheses.

Keywords: Discoloration, Fixed dental prosthesis, Fracture resistance, Marginal adaptation, Metal ceramic, PEEK, Porcelain fused to metal.

World Journal of Dentistry (2022): 10.5005/jp-journals-10015-1882
\end{abstract}

\section{INTRODUCTION}

Fixed partial dentures have been an effective therapeutic option for replacement of missing teeth. Fixed partial dentures (FPDs) have evolved in materials over time. The first documented use of dental materials dates back to 500 BC. Dental fixed prostheses were first made of gold by the Etruscans. Metals like Ni and Cr were also employed to construct the framework for the fixed prosthesis. After that metal frameworks veneered with tooth-colored ceramics were used. ${ }^{1}$ As esthetics began to become the main concern in patient satisfaction, research and development started focusing on metal-free and all-ceramic materials like lithium disilicate and aluminum oxide ceramics. ${ }^{2}$

Frameworks manufactured from pre-sintered oxide ceramics were introduced to dentistry with the advancement of computer-aided design/computer-assisted manufacture (CAD/CAM) milling technology. Because of their low failure rate ( $8 \%$ to $10 \%$ after ten years), metal-ceramic fixed partial dentures are considered the gold standard for posterior FPDs. Alternatives to metal-based restorations were supplied by industrially made ceramic or zirconia frameworks. They are sturdy and structurally sound enabling great fitness for clinical use. The enhanced porcelain chipping rate is the one major disadvantage of the zirconia framework. ${ }^{3}$ In recent years newer materials have been tested to be used in dentistry. One such material is Polyether Ether Ketone (PEEK). ${ }^{4,5}$

Polyether ether ketone is a linear semi-crystalline polymer made via nucleophilic substitution of aromatic dihalides with bisphenol salt. The occurrence of aromatic rings deliberates resistance to mechanical loads, oxidative and thermal assaults. Polyether ether ketone has a high melting point of $342.85^{\circ} \mathrm{C}$, as well as excellent

\begin{abstract}
${ }^{1-3}$ Department of Prosthodontics, Saveetha Dental College and Hospitals, Saveetha Institute of Medical and Technical Sciences, Saveetha University, Chennai, India

Corresponding Author: Dhanraj Ganapathy, Department of Prosthodontics, Saveetha Dental College and Hospitals, Saveetha Institute of Medical and Technical Sciences, Saveetha University, Chennai, India, e-mail: dhanrajmganapathy@yahoo.co.in

How to cite this article: Kamlesh RD, Nallaswamy D, Ganapathy D. Effectiveness of PEEK Framework in Comparison to Metal Framework for Fixed Dental Prosthesis: A Systematic Review. World J Dent 2022;13(1):80-86.
\end{abstract}

Source of support: $\mathrm{Nil}$

Conflict of interest: None

thermal steadiness, chemical resilience, and radiolucency. It is a stiff polymer at room temperature that can be semi-crystalline or amorphous depending on the manufacturing technique used. ${ }^{6}$

Polyether ether ketone has been identified as a viable biomaterial for medical implants such as spinal and cranial implants. Endosseous implants, provisional abutments for implant-supported prostheses, prosthetic substructure over implants, and removable as well as fixed partial dentures are a few applications for PEEK in dentistry.

Polyether ether ketone is a well-proven material with outstanding mechanical qualities, primarily for use in load-bearing areas. It also has a low rate of biofilm formation and, due to its lightweight, provides excellent patient comfort. ${ }^{7}$ Polyether ether ketone is a high-performance polymer that is biologically inert and water-insoluble. Based on its outstanding physical as well as biological characteristics, this material appears to be an

(c) The Author(s). 2022 Open Access This article is distributed under the terms of the Creative Commons Attribution 4.0 International License (https://creativecommons.org/licenses/by-nc/4.0/), which permits unrestricted use, distribution, and non-commercial reproduction in anymedium, provided you give appropriate credit to the original author(s) and the source, provide a link to the Creative Commons license, and indicate if changes were made. The Creative Commons Public Domain Dedication waiver (http://creativecommons.org/publicdomain/zero/1.0/) applies to the data made available in this article, unless otherwise stated. 
appropriate superstructure in dental practice, such as for dental implants, temporary abutments, implant aided bars, framework for removable prostheses, and fixed dental prostheses, despite the paucity of clinical long-term research. ${ }^{8}$ Hence, it is of crucial importance to analyze the literature to explore the role of PEEK in various restorative and prosthetic applications and to validate and compare its clinical effectiveness with respect to metal-ceramic alloy systems in Fixed Dental Prostheses (FDP). This systematic review aims to critically analyze and review various studies that compare PEEK and metal alloys as a framework for a fixed dental prosthesis in terms of fracture resistance, marginal adaptation and discoloration.

\section{Materials and Methods}

This review adhered to the PRISMA guidelines for conducting and reporting systematic reviews and meta-analyses. This systematic review was conducted in the Postgraduate Department of Prosthodontics to analyze and compare the effectiveness of PEEK as a substitute for alloy framework in fixed dental prostheses. Three independent reviewers were involved in the study. They screened the literature pertinent to PEEK in fixed dental prosthetic applications with time limits from the years 1995 January to 2020 January. The search strategy was done based on PICO formulation. PICO analysis was used to create a search questionnaire, and a complete search was piloted in PubMed, Cochrane, Embase, Ovid, Science Direct databases for related manuscripts. The articles for the review were selected based on the inclusion and exclusion criteria. Following screening and final selection a all the relevant information from the articles were extracted, tabulated, analyzed, and interpreted.

\section{Research Question}

Are PEEK frameworks equally effective as metal frameworks in fixed dental prosthetic applications?

\section{Pico Analysis Framework}

A PICO framework was utilized to answer the research question using the following approach elements:

\section{Population (P)}

Partially edentulous arch, Partially edentulous restoration, Partially edentulous rehabilitation, posterior missing teeth, anterior missing teeth, missing tooth, partially edentulous, bilaterally missing teeth.

\section{INTERVENTIONS ( I)}

Polyether Ether Ketone (PEEK), PEEK fixed partial denture, PEEK partial denture prosthesis, PEEK partial dental prosthesis, PEEK crown and bridges, PEEK crowns, PEEK bridges, fixed partial denture, partial denture prosthesis, partial dental prosthesis, crown and bridges bridge, short span bridge, long span bridge, survival of fixed partial dentures.

\section{Comparison (C)}

Cobalt chromium, Nickel chromium, Titanium, Zirconium, CAD-CAM, CAD-CAM zirconium, Computer-aided design, Computer-aided manufacturing, Acrylic, Lithium disilicate ceramics, Layered ceramics, Metal, All metal, All ceramic, Metal ceramic, Porcelain fused to metal, Ceramic facing.

\section{Outcome measures (0)}

Color changes, Marginal adaptation, Marginal fit, Marginal accuracy, Esthetics, Fracture resistance.

\section{Article Eligibility Criteria}

Original research studies on PEEK frameworks and metal-ceramic frameworks for single crowns, fixed partial dentures, and implant fixed partial dentures reporting on the outcome measures were included for the systematic review. Review articles discussing about PEEK frameworks were excluded.

\section{Articles Selection}

The titles and abstracts of the entries found in the first electronic database search results were studied. Following this, full-text copies of potential research that can be included in this review were read, and after applying the eligibility criteria, a final selection of publications was made. The screening process was done by all three independent researchers and the studies were finally consolidated.

\section{Results}

Three independent reviewers were involved in the study and their inter-observer reliability was high with a kappa score of 83 . The reviewers screened the literature and 140 papers were rejected grounded on the title and the abstract from the 165 publications found after scanning all databases. Twenty of the other 25 research were barred based on our inclusion criteria, while five were included based on core data. As shown in the flowchart (Fig. 1), the five articles were examined and consolidated. The treatment outcomes observed in these studies were fracture resistance, aesthetics, and marginal accuracy.

\section{Data Extraction}

Using standardized abstraction tables, the data from the selected studies were retrieved. In one table, the following general features of each study were extracted: (1) Author and year (2) Type of study (3) Groups (4) Sample size (5) Categories of statistical methods used (6) Outcome assessment (7) Scientific method used for assessment (8) Inference. The information was extracted from the studies, consolidated, and analyzed in-depth (Table 1). The Oxford Centre for Evidence-based Medicine's 2011 level of evidence was also tabulated and the risk of bias was high for four in vitro studies and moderate for one randomized trial (Table 2).

\section{Search Process and Article Selection}

This flowchart depicts the overall volume of articles obtained, discarded and the total number of publications retrieved for analysis (Fig. 2).

\section{Discussion}

Fixed dental prostheses (FDPs) have long been the gold standard for replacing lost teeth because they improve patient satisfaction, masticatory capability, and comfort. ${ }^{9}$ In this systematic review, we have explored the literature to review the use of nonmetal alternatives like Polyether ether ketone for a fixed partial denture framework. This systematic review focused on the outcomes of four in vitro studies and one randomized controlled trial for a fixed partial denture framework fabricated by metal and PEEK.

Nazari et al. compared the fracture strength of implantsupported FPDs with excessive crown height made of zirconia, 


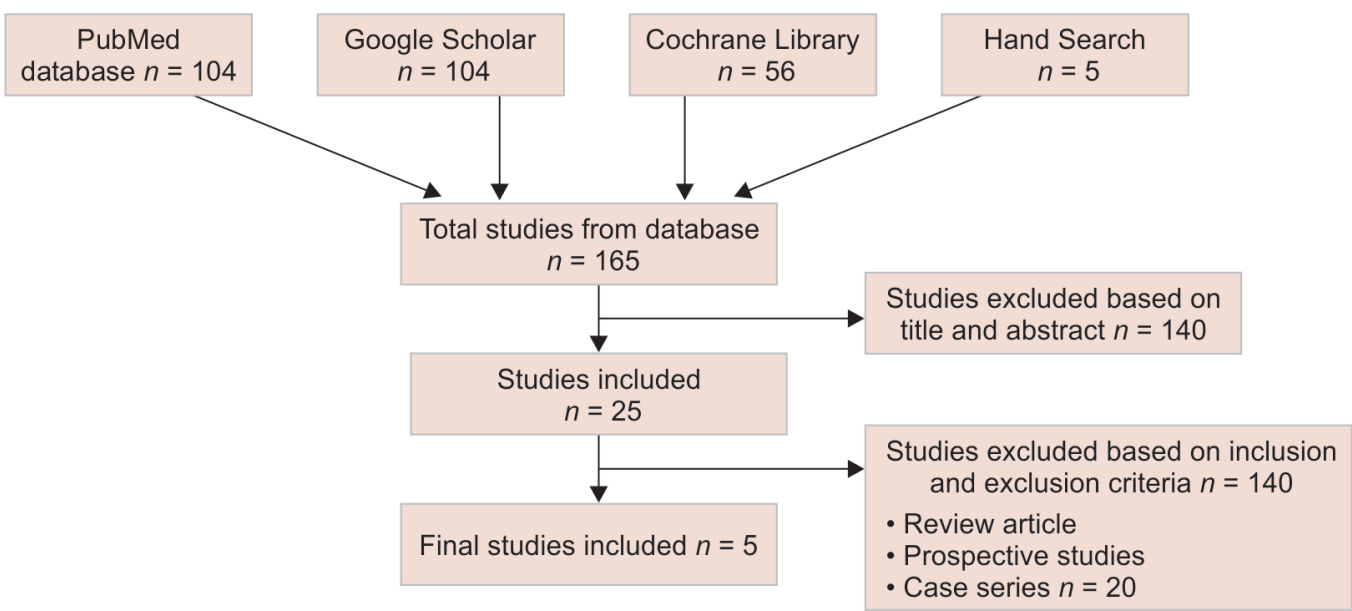

Fig. 1: Depicting the search methodology describing the total number of articles obtained, the ones that were excluded, inclusion of handpicked articles and finally the total number of articles that were retrieved for analysis

nickel-chromium alloy ( $\mathrm{Ni}-\mathrm{Cr}$ ), and PEEK materials and concluded that all implant-supported three-unit FPDs made of zirconia, metal ceramic, and PEEK materials were effective in withstanding the bite force and para-functions ${ }^{7}$ Tekin et al. used finite element analysis to compare the stresses in the peri-implant bone, implants, crowns, abutments, and screws after loading using PEEK materials, which are an alternative to titanium abutment and metal supported restorations, and observed that the PEEK material reduced stress on abutment significantly. ${ }^{10}$

The load usually engaged by the missing tooth must be transferred through the pontic, connectors, and retainers to the periodontium of the abutment teeth. The fracture load of metal, zirconia was similar compared to PEEK framework. As per the results of this research, all the implant-supported three-unit FPDs made of zirconia, metal ceramic, and PEEK components may resist bite force even when para-functions occur in the molar region with excessive crown height space. The stress upon abutment was found to be lower in groups using PEEK crowns as a crown material compared to groups utilizing metal-ceramic crowns. ${ }^{7,10}$

For the success of fixed prostheses, the marginal fit is a critical aspect to consider. Clinically acceptable discrepancies in the range of $40-120 \mu$ are deemed clinically acceptable for crown success. ${ }^{11}$ The marginal gap should be less than 120 microns in order to be considered clinically acceptable. According to studies, the marginal gap for cemented restorations was in the range of 25-40 microns and was regarded a clinically acceptable. ${ }^{12,13}$

Microleakage and secondary cavities, plaque deposition, periodontal inflammation, and bone deterioration are all risks of an incorrect marginal fit, which can shorten the life of prosthetic restorations. ${ }^{14}$ Polyether ether ketone crowns have a substantially greater marginal fit accuracy than PFM crowns. Using a stereomicroscope and $\mathrm{CBCT}$, Roy et al. compared the marginal accuracies of traditional Porcelain Fused to Metal (PFM) and PEEK crowns, concluding that marginal accuracies in PEEK crowns were substantially higher and better than PFM crowns. ${ }^{15}$ As per Nawafleh et al., there was a significant lack of agreement on marginal adaptation of multiple crown systems due to discrepancies in testing methodology and experimental methodologies. ${ }^{16}$

The chromogenic characteristics of PEEK, zirconia $\left(\mathrm{ZrO}_{2}\right)$, and cobalt-chromium-molybdenum alloy ( $\mathrm{CoCrMo}$ ) veneering materials over core materials were examined by Stawarczyk et al. Color was determined with a spectrophotometer (CIE-System) and a chair-side color assessment device (VITA EasyShade) after specimens were superimposed on assemblies. With respect to CIE-LAB System and VITA EasyShade specifications for each veneering material, PEEK as a core material produced equivalent aesthetic results to $\mathrm{ZrO}_{2}$ and $\mathrm{CoCrMo} .{ }^{17}$

The color of the neighboring teeth, optical qualities such as scattering, absorption, and reflection, and intrinsic component feature such as opacity and translucency of the restoration all influence the eventual appearance and awareness of dental restorations. Polyether ether ketone frameworks, relative to metal frameworks, offer greater aesthetics, according to studies. With respect to the CIELAB System characteristics, PEEK as a core material revealed no different trends when equated to the gold standard core materials such as $\mathrm{ZrO}_{2}$. The core materials and the change between the framework and veneering material, on the other hand, had a substantial impact on the CIELAB System and VITA Easy Shade results, indicating that the framework and veneering material combination was important. ${ }^{17}$

Fracture resistance during mechanical loading is a crucial factor in determining longevity and success in prosthetic frameworks. ${ }^{18}$ Heloisa et al. employed the finite element method to evaluate the biomechanical behavior of tooth-supported fixed partial prosthesis components made of $\mathrm{Cr}$-Co alloy and PEEK when subjected to physiological occlusal loads and found that the two materials had similar outcomes. ${ }^{19}$

This systematic review appraises both the features of PEEK and different alternatives of metal in the fabrication of fixed partial dentures, thus providing a better understanding of the clinical and mechanical properties of the metal and nonmetal (PEEK) framework for fixed dental prostheses. This will enable the clinician to make better treatment choices. ${ }^{18,19}$ It must be observed that the literature search was steered principally using electronic databases and limited to reviewing references of included studies, hence the current review may not have acknowledged all the nuances in literature. The level of evidence of this systematic review is not very high due to the paucity of studies and the risk of bias is high for the selected studies. Another observation of this review is the selected articles did not evaluate gingival and periodontal health as an outcome measure. Furthermore, meta-analysis was not applicable due to heterogeneity among the studies. Hence, there is a need for future studies evaluating marginal accuracy, aesthetics, and gingival health with long-term follow-up to shed more light on these criteria. 


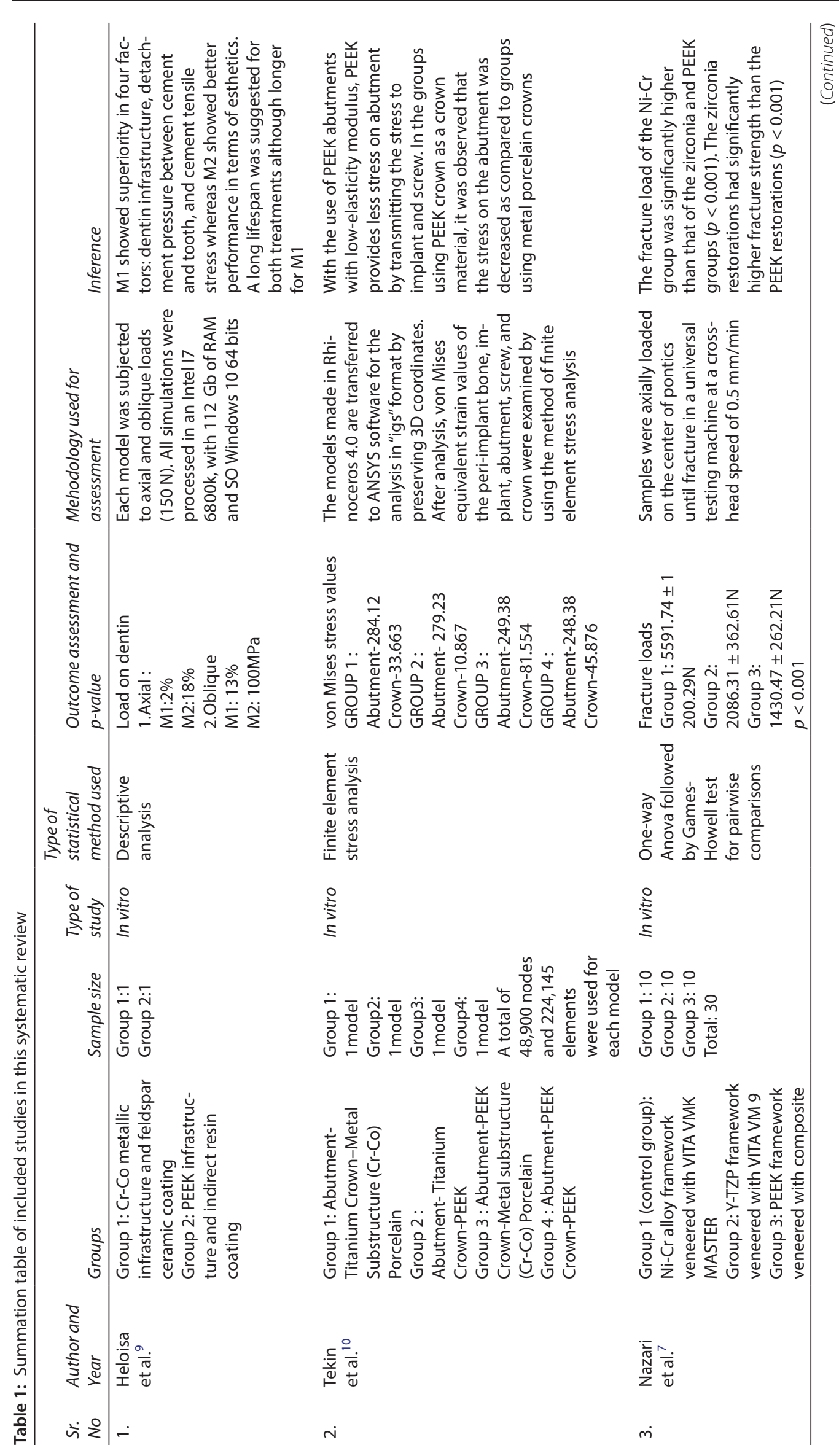




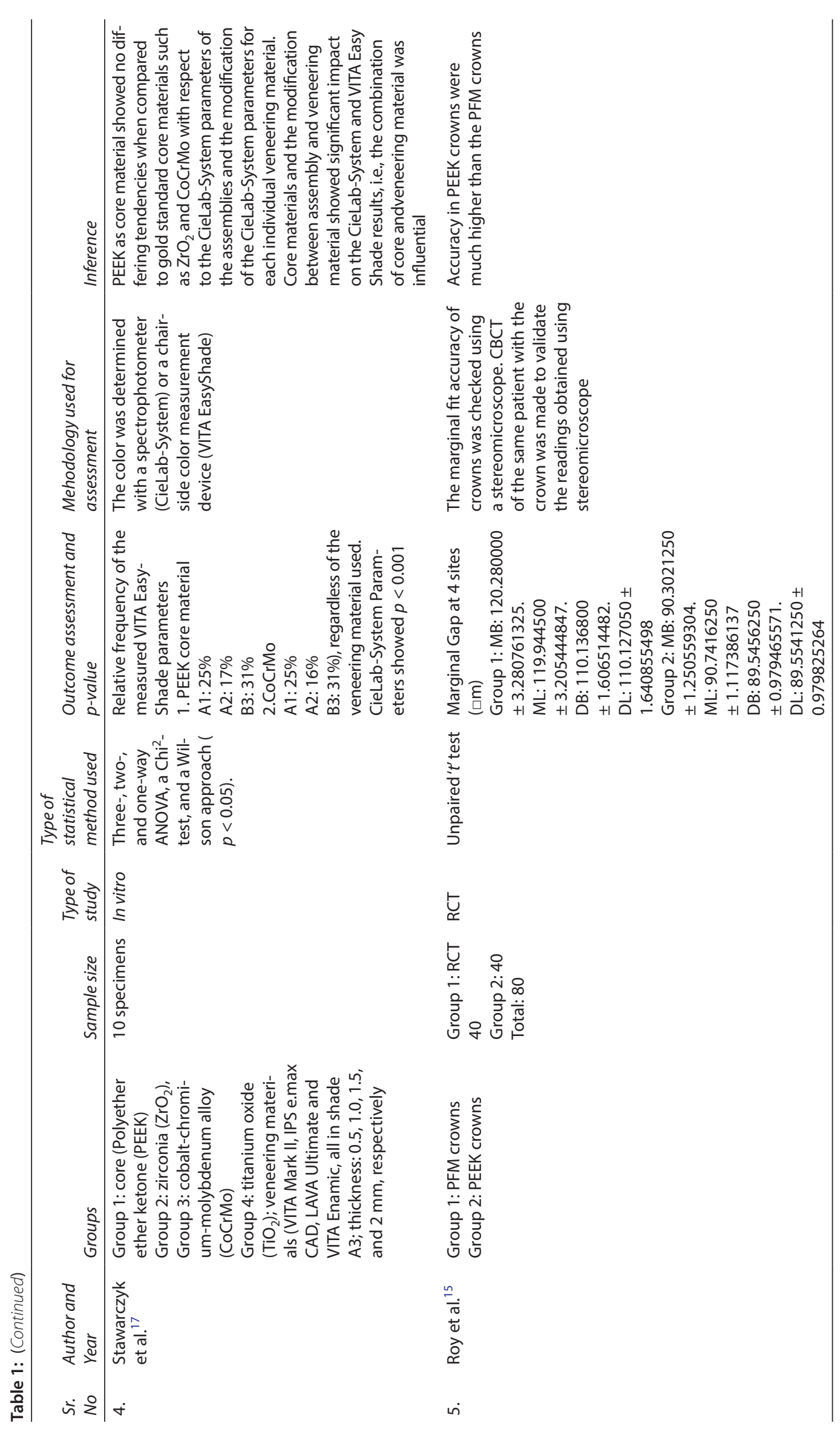


Effectiveness of PEEK Framework for Fixed Dental Prosthesis

Table 2: CEBM level of evidence of included studies (According to Oxford Centre for Evidence-based Medicine 2016, levels of evidence)

\begin{tabular}{lllll}
\hline Sr. No & Author and year & Study design & Level of evidence & Risk of bias \\
\hline 1. & Heloisa et al. ${ }^{19}$ & In vitro study & Level 5 & High \\
2. & Tekin et al. ${ }^{20}$ & In vitro study & Level 5 & High \\
3. & Nazari et al. ${ }^{21}$ & In vitro study & Level 5 & High \\
4. & Stawarczyk et al. ${ }^{22}$ & In vitro study & Level 5 & High \\
5. & Roy et al. $^{23}$ & RCT & Level $1 \mathrm{~b}$ & Moderate \\
\hline
\end{tabular}

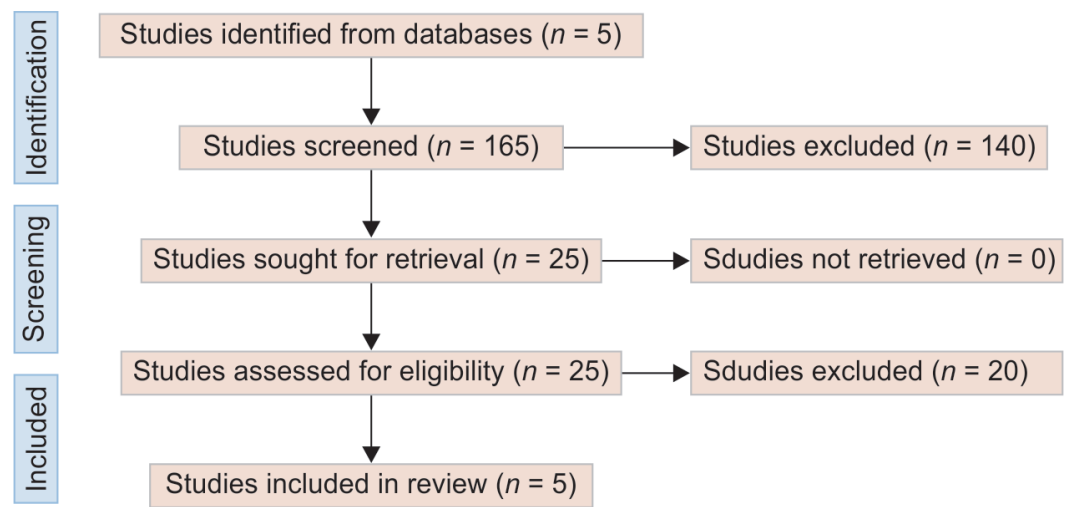

Fig. 2: PRISMA flowchart for selection of articles

\section{ConcLusion}

Based on this systematic review, PEEK fixed partial dentures are reported to show better esthetics and despite the fact, PEEK frameworks showed equivalent mechanical properties as metal alloys, there is in sufficient irrefutable evidence to validate superior mechanical properties compared to its metal counterpart. Hence, future studies are required in this field for further understanding and authentication. Nevertheless, PEEK can be effectively used as an appropriate superstructure for dental implants, temporary abutments, implant aided bars, connectors for removable prostheses, and fixed dental prostheses.

\section{Authors Contribution}

All authors were involved in data collection, drafting the manuscript, revising the manuscript, and had approved the submission of the manuscript.

\section{References}

1. Svanborg P, Stenport V, Eliasson A. Fit of cobalt-chromium implant frameworks before and after ceramic veneering in comparison with CNC-milled titanium frameworks. Clin Exp Dent Res 2015;1(2):49-56. DOI: $10.1002 / \mathrm{cre} 2.9$

2. Jokstad A, Gökçe M, Hjortsjö C. A systematic review of the scientific documentation of fixed partial dentures made from fiber-reinforced polymer to replace missing teeth. Int JProsthodont 2005;18(6):489-496. DOI:10.1016/j.prosdent.2006.05.021

3. Yoshida K, Tsuo Y, Atsuta M. Bonding of dual-cured resin cement to zirconia ceramic using phosphate acid ester monomer and zirconate coupler. J Biomed Mater Res B Appl Biomater 2006;77(1):28-33. DOI: $10.1002 / \mathrm{jbm} \cdot$ b. 30424

4. Kurtz SM, Devine JN. PEEK biomaterials in trauma, orthopedic, and spinal implants. Biomaterials 2007;28(32):4845-4869. DOI: 10.1016/j. biomaterials.2007.07.013

5. Skirbutis G, Dzingutè $A$, Masiliūnaitè $V$, et al. A review of PEEK polymer's properties and its use in prosthodontics. Stomatologija 2017;19(1):19-23.
6. Pascual A, Toma M, Tsotra P, et al. On the stability of PEEK for short processing cycles at high temperatures and oxygen-containing atmosphere. Polym Degrad Stab 2019;165:161-169. DOI: 10.1016/j. polymdegradstab.2019.04.025

7. Nazari V, Ghodsi S, Alikhasi M, et al. Fracture strength of three-unit implant supported fixed partial dentures with excessive crown height fabricated from different materials. J Dent 2016;13(6): 400-406.

8. Stawarczyk B, Beuer F, Wimmer T, et al. Poly Ether Ether ketone-a suitable material for fixed dental prostheses? J Biomed Mater Res Part B Appl Biomater 2013;101(7):1209-1216. DOI: 10.1002/jbm.b.32932

9. Pjetursson BE, Sailer I, Makarov NA, et al. All-ceramic or metal-ceramic tooth-supported fixed dental prostheses (FDPs)? A systematic review of the survival and complication rates. Part II: multiple-unit FDPs. Dent Mater 2015;31(6):624-639. DOI: 10.1016/j.dental.2015.02.013

10. Tekin S, Değer Y, Demirci F. Evaluation of the use of PEEK material in implant-supported fixed restorations by finite element analysis. Niger J Clin Pract 2019;22(9):1252-1258. DOI: 10.4103/njcp.njcp_144_19

11. Goldin EB, Boyd NW , Goldstein GR, et al. Marginal fit of leucite-glass pressable ceramic restorations and ceramic-pressed-to-metal restorations. J Prosthet Dent 2005;93(2):143-147. DOI: 10.1016/j. prosdent.2004.10.023

12. Pera $P$, Gilodi $S$, Bassi $F$, et al. In vitro marginal adaptation of alumina porcelain ceramic crowns. J Prosthet Dent 1994;72(6):585-590. DOI: 10.1016/0022-3913(94)90289-5

13. Schlenz MA, Vogler JAH, Schmidt A, et al. Chairside measurement of the marginal and internal fit of crowns: a new intraoral scan-based approach. Clin Oral Investig 2020;24(7):2459-2468. DOI: 10.1007/s00784-019-03108-3

14. Schlenz MA, Schmidt A, Wöstmann B, et al. In vitro comparison of analog versus digital impressions of the periodontally compromised dentition focused on interdental areas. Int J Comput Dent 2019;22(2):131-138.

15. Roy MS, Tewary S, Sanyal P, et al. An in vivo study to compare the marginal fit accuracy of crowns prepared using two different materials. J Evol Med Dent Sci 2019;8(24):1930-1934. DOI: $10.14260 /$ jemds/2019/424

16. Nawafleh NA, Mack F, Evans J, et al. Accuracy and reliability of methods to measure marginal adaptation of crowns and FDPs: a literature review. J Prosthodont 2013;22(5):419-428. DOI: 10.1111/jopr.12006 
17. Zoidis P, Papathanasioul, Polyzois G. The use of a modified Poly-Ether-Ether-Ketone (PEEK) as an alternative framework material for removable dental prostheses. A clinical report. J Prosthodont 2016;25(7):580-584. DOI: 10.1111/jopr.12325

18. Stawarczyk B, Schmid P, Roos M, et al. Spectrophotometric evaluation of Poly Ether Ether ketone (PEEK) as a core material and a comparison with gold standard core materials. Materials 2016;9(6):491-501. DOI: 10.3390/ma9060491
19. Santos Heloisa RB, Ávila Gisseli B, Carvalho Geraldo AP, et al. Biomechanical behavior of tooth-supported fixed partial prostheses components with two different infrastructures: metal and Polyether Ether ketone (PEEK). Oral Health Dent Manag 2019;18(3):1-5. DOI: 10.35248/2247-2452.19.18.1074 